\title{
Hybrid Partial Least Squares models for batch processes: Integrating data with process knowledge
}

\author{
Debanjan Ghosh ${ }^{1}$, John F MacGregor ${ }^{1,2}$, and Prashant Mhaskar ${ }^{1}$ \\ ${ }^{1}$ Department of Chemical Engineering, McMaster University, Hamilton, Ontario, \\ Canada, $L 8 S 4 L 7$ \\ ${ }^{2}$ ProSensus Inc., Burlington, Ontario, Canada \\ ${ }^{1}$ Email : mhaskar@mcmaster.ca \\ ${ }^{2}$ Email : john.macgregor@prosensus.ca
}




\section{Supporting Information for Publication}

Table S1: Seeded batch crystallizer parameters

\begin{tabular}{ll}
\hline \multicolumn{2}{c}{ Parameter nomenclature } \\
\hline$C:$ Solute concentration & $T:$ Reaction temperature \\
$C_{m}:$ Metastable concentration & $C_{s}$ : Saturation concentration \\
$\mu_{i}:$ ith moment of the particle size distribution & $T_{j k}$ : Jacket temperature \\
$\mu_{i}^{n}:$ ith moment corresponding to nucleation & $\rho:$ Density of crystals \\
$\mu_{i}^{s}:$ ith moment corresponding to seed/desired & $k_{v}:$ Volumetric shape factor \\
$U:$ Overall heat transfer co-efficient & $A:$ Total heat transfer surface area \\
$M:$ Mass of solvent in crystallizer & $C_{p}:$ Heat capacity of solution \\
$\Delta H:$ Heat of reaction & $E_{b}:$ Nucleation activation energy \\
$E_{g}:$ Growth activation energy & \\
$b:$ exponent relating nucleation rate to supersaturation & \\
$g:$ exponent relating growth rate to supersaturation & \\
\hline
\end{tabular}

Table S2: Parameter values of the batch crystallizer and simulation settings

\begin{tabular}{ll}
\hline Parameters & Nominal Values \\
\hline$b$ & 1.45 \\
$g$ & 1.5 \\
$k_{b}$ & $285 \mathrm{~s}^{-1} \mu \mathrm{m}^{-3}$ \\
$k_{g}$ & $1.44 \mathrm{e} 8 \mu \mathrm{m} / \mathrm{s}$ \\
$\rho$ & $2.66 \mathrm{e}-12 \mathrm{~g} / \mathrm{m}^{3}$ \\
$U A$ & $0.8 \mathrm{KJ} \mathrm{s} \mathrm{K}$ \\
$k_{v}$ & 1.5 \\
$C_{p}$ & $3.8 \mathrm{KJ} /(\mathrm{Kg} \mathrm{K})$ \\
$\Delta H$ & $44.5 \mathrm{KJ} / \mathrm{Kg}$ \\
$E_{b} / R$ & $7517 \mathrm{~K}$ \\
$E_{g} / R$ & $4859 \mathrm{~K}$ \\
$M$ & 27 \\
$d t$ & $6.01 \mathrm{~seconds}$ \\
$T(0)$ & $50^{\circ} \mathrm{C}$ \\
$C(0)$ & $0.1742 \mathrm{~g} / \mathrm{g}$ \\
\hline
\end{tabular}


Table S3: Percentage error in the parameters of the first principles model (original values of these parameters are listed in Table S2).

\begin{tabular}{ccc}
\hline \multirow{2}{*}{ Parameter } & \multicolumn{2}{c}{$\%$ Mismatch } \\
\cline { 2 - 3 } & Case-1 & Case-2 \\
\hline$E_{b} / R$ & 0.8 & 0.8 \\
$E_{g} / R$ & 0.8 & 0.8 \\
$g$ & -1 & 1 \\
$b$ & -1 & 1 \\
$k_{b}$ & 5 & 5 \\
$k_{g}$ & 5 & 5 \\
$k_{v}$ & -30 & -10 \\
$\Delta H$ & -30 & -10 \\
\hline
\end{tabular}

Table S4: Batch initial conditions and noise parameters

\begin{tabular}{ccc}
\hline Variable & $\sigma_{\text {batchwise }}$ & $\sigma_{\text {noise }}$ \\
\hline$n$ & 0.2 & 0.05 \\
$C$ & 0.0028 & 0.008 \\
$T$ & 0.52 & 0.003 \\
$\mu_{i}^{n}, \mu_{i}^{s}(i=1,2,3)$ & - & 0.001 \\
\hline
\end{tabular}

Table S5: PI controller settings used in the identification and validation batches

\begin{tabular}{cc}
\hline Input policy & Number of Batches \\
\hline PI trajectory tracking $\left(K_{C}=0.85, T_{I}=38\right)$ & 50 \\
\hline
\end{tabular}

Table S6: Tuning parameters of Kalman filter used in the estimation of terminal subspace states

\begin{tabular}{cclc}
\hline Model & Process noise $\left(\sigma^{2}\right)$ & Measurement noise $\left(\sigma^{2}\right)$ \\
\hline Subspace & 0.001 & 0.0004 & 0.0004 \\
\hline
\end{tabular}




\begin{tabular}{c|c|c|c|c|c|c|c|c|c|c|c|}
\multicolumn{9}{c}{$\begin{array}{c}\text { Process } \\
\text { measurements }\end{array}$} & \multicolumn{8}{c}{$\begin{array}{c}\text { State trajectory data from modified first } \\
\text { principles model }\end{array}$} \\
\hline Obs ID & C & $\mathrm{T}$ & $\mathrm{T}_{\mathrm{j}}$ & $\mu_{0}^{n}$ & $\ldots$ & $\mu_{3}^{n}$ & $\mu_{0}^{s}$ & $\ldots$ & $\mu_{3}^{s}$ & $\mathrm{C}_{\mathrm{s}}$ & $\mathrm{C}_{\mathrm{m}}$ \\
\hline 1 & & & & & & & & & & & \\
\hline 2 & & & & & & & & & & & \\
\hline$\vdots$ & & & & & & & & & & & \\
\hline $\mathrm{N}$ & & & & & & & & & & & \\
\hline
\end{tabular}

Figure S1: X block of Augmented/Hybrid PLS for the crystallizer case study 